\title{
Sobreviviendo al margen. Intercambio y redes de reciprocidad entre hogares en situación de pobreza de Villa Lugano
}

\author{
Surviving on the fringe. Exchange and reciprocity networks among \\ impoverished households in Villa Lugano
}

\section{Helga Fourcade}

Observatorio de la Deuda Social Argentina. Pontificia Universidad Católica Argentina

Resumen

\begin{abstract}
El artículo presenta una investigación, desde la perspectiva de los actores, del funcionamiento de las redes de intercambio recíproco entre un grupo de hogares en situación de pobreza de Villa Lugano (Buenos Aires, Argentina). Para su abordaje fue seleccionado un enfoque analítico basado en los activos, la vulnerabilidad y la estructura de oportunidades. En ese marco, se pretende analizar las modificaciones en el abanico de activos del que disponen los hogares, ante las variaciones de los elementos constitutivos de la estructura de oportunidades en la que se desenvuelven, haciendo énfasis en las características particulares que determinan su lugar en las redes de las que participan. El mercado de trabajo, el Estado y la sociedad representan tres escenarios que, cada uno con sus particularidades, influyen considerablemente en las capacidades de los hogares a la hora de transformar los recursos en activos, condicionando las adaptaciones de los hogares en vistas de mantener o incrementar su bienestar.
\end{abstract}

Palabras Clave: Redes de Reciprocidad; Activos; Vulnerabilidad; Estructura de Oportunidades; Programas de Transferencias de Ingreso Condicionadas

\begin{abstract}
This article presents a research from the perspective of the actors, the operation of reciprocal exchange networks among impoverished households in Villa Lugano (Buenos Aires, Argentina). The framework selected for its analytical approach is based on assets, vulnerability and opportunities structure. We intend to analyze the changes in the portfolio of assets available to households; how they adapt when faced with changes in the components of the opportunities structure, focusing on the particular characteristics that determine the household's place within the network. The labor market, the state and society, each with its own particularities, represent three settings that can drastically affect a household's capacity to transform their resources into assets and ultimately determine the adjustments it must make in order to either maintain or improve their overall welfare.
\end{abstract}

Keywords: Reciprocity Networks; Assets; Vulnerability; Opportunities Structure; Conditional Cash Transfers Programs

\section{Introducción}

Una de las problemáticas más trabajadas a finales de los sesentas y comienzos de los setentas sobre la pobreza urbana puede resumirse en el título del famoso libro escrito por Larissa Lomnitz “¿Cómo sobreviven los marginados?" (1975). Allí se explicaba, que los pobres tenían formas o "estrategias" de supervivencia que les eran propias, basadas en 
redes de intercambio recíproco articuladas entre familiares, amigos y vecinos, de forma tal que generaban una suerte de sistema de seguridad social informal. El intercambio de bienes y servicios, enmarcado en el entrelazamiento de estas redes, está determinado por las características particulares de cada uno de los actores participantes, en este caso los hogares, en relación a su capacidad de transformar los recursos de los que disponen en activos, en vistas de mantener o incrementar su bienestar.

Asimismo, un enfoque desarrollado en la última década por un grupo de investigadores de la CEPAL Montevideo (Filgueira \& Katzman 1999), permite analizar a los hogares, tanto en su integralidad como en su particularidad, el funcionamiento de la "caja negra" al interior de los hogares, en el proceso de transformación de recursos en activos. La mirada integral del enfoque está dada a través de un concepto que denominan "estructura de oportunidades" - en la que se desenvuelven los hogares-, y la mirada particular está centrada en cómo son afectados los hogares, con las variaciones de dicha estructura en el proceso de transformación de los recursos en 'activos'.

Los Programas de Transferencias de Ingreso Condicionadas (en adelante PTC) forman parte de una generación de programas sociales de desarrollo, que promueven la acumulación de capital humano de los hogares a los que benefician, con el fin de interrumpir con el ciclo de reproducción intergeneracional de la pobreza. Brindan un incentivo monetario, determinan su población objetivo a través de un mecanismo de focalización y, como su nombre lo indica, cuentan con condicionalidades.

Este artículo se basa en el análisis de las redes de intercambio recíproco de las que participan un grupo de hogares de Villa Lugano (Ciudad de Buenos Aires), desde un abordaje multidimensional a través de la aplicación del enfoque de Activos, Vulnerabilidad y Estructura de Oportunidades (en adelante AVEO) destacando la perspectiva de los actores ante las variaciones de los elementos constitutivos de la estructura de oportunidades, principalmente aquellas originadas por la transferencia del programa Ciudadanía Porteña.

El enfoque AVEO que busca demostrar las potencialidades de un abordaje centrado en los recursos que pueden movilizar los hogares, sin limitarlo a la noción de capital en términos exclusivamente económicos o monetarios. Se basa principalmente en los tres conceptos que constituyen su nombre: activos, estructura de oportunidades y vulnerabilidad. He aquí una breve definición de cada uno de ellos.

Por 'activos' se entiende el conjunto de recursos, materiales e inmateriales que los hogares movilizan en pos de mejorar su desempeño económico y social, así como también, como recursos desplegados para evitar el deterioro de sus condiciones de vida o disminuir su vulnerabilidad (Filgueira \& Katzman 1999). En lo que respecta a la 'estructura de oportunidades', ésta puede ser analizada, al menos, desde tres escenarios: el mercado, el Estado y la sociedad. El mercado ha sido considerado tradicionalmente como la principal estructura de oportunidades. Sus variaciones y transformaciones en la estructura productiva, son factores que modifican la estructura e inciden sobre las posibilidades diferenciales de individuos y hogares. El Estado -dependiendo de la matriz institucional de cada país- tiene un papel central en la conformación de las oportunidades, a través de cuyo impacto directo e indirecto, inciden sobre la estructura. Los diferentes regímenes de 'Estado de Bienestar' con su potencial efecto distributivo inciden en las oportunidades mediante procesos de transferencia entre sectores y grupos que se canalizan en el ofrecimiento de bienes y servicios. Y por último, las diferentes formas de asociabilidad, modalidades de acción colectiva, organización y peso de la comunidad y la familia, capital social, redes de interacción, tendencias demográficas, y en general, los cambios en las instituciones primordiales de la sociedad, pueden ser señaladas como fuentes de alteración de la estructura de oportunidades (Filgueira 2001). 
Por su parte, la 'vulnerabilidad' es entendida como una relación entre los dos términos señalados hasta aquí: los 'activos' y la 'estructura de oportunidades'. Esto se debe a que de las diferentes combinaciones entre ambos términos, se derivan distintos tipos y grados de vulnerabilidad (Filgueira \& Katzman 1999: 8).

En ese marco, existe un concepto adicional que debe ser incorporado al análisis. Se trata del concepto de 'hogar'. Teniendo en cuenta que la unidad de análisis utilizada para el abordaje son los hogares, se tomará en cuenta a Mercedes González de la Rocha, quien denominó al hogar como "unidad doméstica urbana". Este concepto refiere al grupo de gente que vive bajo el mismo techo, organiza sus recursos colectivamente y pone en acción estrategias de generación de ingresos y actividades de consumo - incluyendo a los miembros que pueden o no ser parientes- (González de la Rocha 1986).

Es bajo este paraguas conceptual, se analizan las modificaciones en la estructura de oportunidades de los hogares, principalmente aquellas originadas por las transferencias de ingreso recibidas a partir del PTC Ciudadanía Porteña.

Para el análisis fueron seleccionados los hogares que eran beneficiarios del programa del barrio porteño de Villa Lugano. Al interior del mismo, fueron seleccionados como área de intervención a tres 'sub- barrios': Piedrabuena, Pirelli y la villa 20. El trabajo de campo fue realizado durante los años 2008 y 2009 a través de visitas intermitentes a los tres subbarrios seleccionados de Villa Lugano.

\section{La estructura de oportunidades y el mercado de trabajo}

Sobre la base de los trabajos de Caroline Moser (1996) y Jeremy Holland (Moser \& Holland 1997) acerca del asset vulnerability framework publicados en el marco institucional del Banco Mundial, se desarrolló la perspectiva latinoamericana, a partir de la línea de estudios de estructuras de oportunidades - activos y vulnerabilidad desarrollada por Filgueira (1998); Filgueira y Katzman (1999) y Katzman et al. (1998), desde la sede de Montevideo de la CEPAL.

El marco conceptual que proponen busca explicar las diferencias entre la vulnerabilidad, la pobreza y la exclusión social, de distintas categorías de hogares urbanos. Esta perspectiva privilegia como factor explicativo, por un lado, el grado de ajuste entre los activos que pueden movilizar los hogares, y por el otro, los requerimientos de acceso a las fuentes de renovación y acumulación de los activos necesarios para participar plenamente en la sociedad. El conjunto de esas fuentes de activos constituye lo que denominan la "estructura de oportunidades" (Katzman \& Filgueira 2006).

Tanto el asset vulnerability framework (Banco Mundial) como el AVEO (CEPAL), parten de la existencia de activos, refiriendo a la posesión, movilización y control de recursos materiales, que permiten a los individuos desenvolverse en la sociedad. Algunas ejemplificaciones de los mismos son el capital financiero, capital humano, experiencia laboral, educación, composición y atributos de la familia, participación en redes, etc. (Filgueira 2001).

Katzman et al. (1998), asumen la siguiente clasificación de los activos basada en los tipos de capital - las formas de los mismos- , para luego enfatizar en las fuentes de estos activos y el proceso de transformación en otros, construir la base de otros, o fundirse entre sí. El modelo que plantean propone tres tipos de activos básicos: (a) capital físico, que constituye uno de los medios fundamentales de acceso al bienestar básico, (b) el capital humano, vinculado principalmente al acceso al trabajo y la autoempleo, y por último (c) 
capital social, directamente relacionado a la propia red de relaciones que define la forma de capital, basado en la confianza y la reciprocidad ${ }^{1}$.

El mercado es una de las esferas fundamentales en las cuales los activos son generados, apropiados y utilizados, brindando oportunidades a través de diversas estructuras de consumo. Entre ellos, se destaca el mercado de trabajo que constituye, en este sentido, un elemento de la estructura de oportunidades fundamental para los hogares.

El empleo, su distribución, su retribución y su estabilidad varían en la historia y la geografía de los países afectando en forma determinante, la suerte de los sectores populares e influyendo al limitar o expandir el repertorio de adaptaciones de estos sectores para realizar trabajo remunerado. Las probabilidades de acceder al empleo o de poder realizar trabajo remunerado dependen, sin duda de los atributos individuales en capital humano, pero también de las características estructurales de la economía y su mercado de trabajo. En este último sentido, el mercado de empleo forma parte de la estructura de oportunidades. (Katzman et al. 1998: 12)

El mercado, y específicamente el mercado de trabajo, es el principal asignador de los recursos que disponen los hogares, por lo tanto, resulta oportuno analizar los efectos de los cambios macro económicos en la organización social de las unidades domésticas, especialmente el impacto de la exclusión laboral.

\subsection{Caracterización del mercado de trabajo en la ciudad}

En la ciudad de Buenos Aires, la situación del mercado laboral se encuentra polarizada entre los grupos poblacionales: 'pobres' y 'no pobres' (determinados en base a la Línea de Pobreza), mostrando muy altos niveles de desempleo en el grupo poblacional pobre. Un informe publicado por el Gobierno de la Ciudad de Buenos Aires (UIMyE 2009) - calculado sobre la Encuesta Anual de Hogares, 2008- expresa que los hogares en situación de pobreza registran tasas de actividad $(53,9)$ y empleo $(38,3)$ más bajas, junto con elevado nivel de desempleo. El análisis de la inserción laboral de esta población evidencia situaciones claramente diferenciadas entre pobres y no pobres. Es destacable el alto nivel de desempleo en la población pobre (29\%).

Las condiciones del mercado de trabajo reducen la capacidad de las unidades domésticas de actuar y responder en la forma 'tradicionalmente conocida', es decir la intensificación en el uso de la fuerza de trabajo familiar para generar la sobrevivencia y reproducción. Los hogares se adaptan al empeoramiento de las condiciones laborales con esfuerzos adicionales. Cuando los ingresos disminuyen, los hogares responden a la situación colocando más miembros en el mercado laboral y aumentando la cantidad de trabajo informal familiar para obtener ingresos en efectivo.

En este sentido, Mercedes González de la Rocha y Agustín Escobar Latapí (2006) sostienen que el deterioro económico, la exclusión laboral y la creciente precariedad del empleo

\footnotetext{
${ }^{1}$ El capital físico está dividido en dos categorías: por un lado, el capital financiero -ahorro monetario, rentas y créditosde alta liquidez, constituye uno de los medios fundamentales de acceso al bienestar básico; y por otro, el capital físico vivienda, animales, maquinarias, medio propios de transporte, etc.-, de menor liquidez que el anterior, aunque cuenta con un potencial de uso casi tan amplio como el capital financiero. El capital humano-que incluye al trabajo como activo principal de los pobres y el valor agregado al mismo en inversiones de salud y educación- es de menor liquidez que los anteriores. Su uso está centrado en el acceso al empleo y al autoempleo remunerado, lo cual constituye una fuente adicional de múltiples tipos de activos. Por último, el capital social -redes de reciprocidad, confianza, contactos, acceso a información- es el menos alienable de todos y sus usos se encuentran fuertemente vinculados a la propia red de relaciones que define dicha forma de capital. Basado en la confianza y la reciprocidad a nivel del intercambio de bienes y servicios no comodificables. (Katzman et al. 1998)
} 
gestan un proceso de erosión de los sistemas sociales de apoyo y de intercambio social, no como resultado inherente de los pobres para sobrevivir y para escapar de su pobreza, sino como el resultado de la pobreza persistente y creciente causada por el deterioro sostenido de los mercados de trabajo.

Las condiciones estructurales de los hogares, varían de acuerdo a la situación particular de cada uno ante la crisis económica y sus efectos en el mercado laboral, teniendo en cuenta que, en Argentina, la crisis económica fue en el año 2001 pero tuvo fuertes consecuencias a lo largo de los años posteriores.

La ausencia de empleo imprime su marca como elemento disruptor en las economías de los hogares y en las formas de organización social al interior de los mismos. Dado que el trabajo es el recurso más importante de los grupos domésticos más vulnerables (Moser 1996; González de la Rocha 1986), "podemos esperar que la exclusión produzca efectos drásticos en la capacidad de los pobres de sobrevivir y reproducirse" (González de la Rocha \& Escobar Latapí 2006: 258).

Al interior de los hogares, Elizabeth Jelín (1984) describe que hay dos tipos de decisiones que constituyen el eje de la organización doméstica. En primer lugar, la decisión de cuándo y cuánto puede y debe trabajar cada miembro, es decir, quiénes y en qué momento van a contribuir a las actividades de mantenimiento de la unidad; $y$, en segundo lugar, la decisión sobre la asignación de dicha capacidad de trabajo a la obtención de ingresos o a la producción doméstica. Estas decisiones, están condicionadas por un factor determinado en las capacidades de los hogares, principalmente vinculado al mercado de trabajo: la fase del ciclo doméstico en la que se encuentren las unidades domésticas.

\subsection{El ciclo doméstico}

Los hogares son unidades muy dinámicas, que evolucionan con el tiempo y se ven expuestas a diversos niveles de vulnerabilidad en el transcurso de las distintas fases del ciclo doméstico por las que van atravesando. Para analizar la naturaleza cambiante de los hogares es necesario adoptar una perspectiva que tenga en cuenta las etapas del proceso de desarrollo de los hogares. La misma se divide en tres fases y cada una de ellas hace una gran diferencia en las capacidades de las unidades domésticas, principalmente en términos de capital humano.

La primera de ellas es la denominada 'fase expansión', que se inicia con la formación cuando se conforma la pareja- y se extiende hasta el momento en que la madre de la familia llega a los 40 años. La edad reproductiva de la mujer es el principal elemento para definir la duración 'la fase expansiva'. La segunda fase es la de 'consolidación', que se inicia al final de la etapa expansiva. Esta puede llamarse igualmente 'fase de equilibrio' por que corresponde a la época en que los hijos se vuelven miembros activos dentro de la estrategia generadora de ingreso, ya no son consumidores, sino también generadores de ingresos. La tercera fase es la de 'dispersión', corresponde a la época del ciclo vital de la unidad doméstica en que los jóvenes abandonan el hogar paterno para poner casa aparte hasta que, finalmente, la unidad doméstica se disuelve (González de la Rocha 1986).

Empero, es importante destacar que las etapas del ciclo doméstico son construcciones analíticas que permiten analizar la naturaleza dinámica de la vida doméstica a lo largo del tiempo, y de ninguna manera son parte de un modelo evolutivo unilateral del cambio doméstico ni cajones perfectamente separables entre sí (González de la Rocha \& Escobar Latapí 2006: 75). Las tres categorías analíticas constituyen "tipos ideales" en términos weberianos, siendo las situaciones más comunes los puntos intermedios y combinaciones inusuales en las dinámicas de los hogares, determinadas por sus características intrínsecas. 


\section{El Estado y la política social}

En Argentina, las políticas focalizadas alcanzaron en la década del noventa un elevado nivel de institucionalización y centralidad entre las políticas sociales estatales. En 1994 se creó la secretaría de Desarrollo Social (SDS), principal organismo para el diseño e implementación de las políticas focalizadas contra la pobreza. "En 1995, la Secretaría de Desarrollo Social puso en marcha el Plan Social del Gobierno Nacional, que tenía por objetivo realizar un ejercicio de planificación estatal del gasto público social" (Isuani 2008: 178).

\subsection{Los Programas de Transferencias de Ingreso Condicionadas (PTC)}

A comienzos del 2002 comenzó en Argentina una nueva manera de pensar las intervenciones sociales por el Estado argentino y ello se debe fundamentalmente a la implementación del 'Programa Jefes y Jefas de Hogar Desocupados' (PJJHD), entre otras medidas. Este programa significó un quiebre radical en la política de protección social. "En unos pocos meses se expandió hasta alcanzar casi tantos beneficiarios como las jubilaciones" (Moreno 2008: 273).

Los PTCs habían emergido con fuerza en la década de 1990, como una nueva herramienta para combatir la pobreza en los países de Latinoamérica en el marco de la crisis del Estado de Bienestar (Bastagli 2011). Desde el comienzo, tuvieron como principal objetivo promover el desarrollo humano a través de la entrega de incentivos monetarios a familias de bajos ingresos, bajo la exigencia de cumplir ciertas condicionalidades en las áreas de salud y educación especialmente por parte de los niños y jóvenes del hogar.

Constituyen una modalidad relativamente nueva en la política social argentina. Durante el siglo XX, el cúmulo de políticas de protección social se estructuró, generalmente, alrededor del empleo formal y del sistema de seguridad social, de larga data y cobertura relativamente alta en el contexto de América Latina. Los trabajadores registrados accedían a beneficios contributivos, como los provisionales y las asignaciones familiares. Otras transferencias monetarias directas, como las pensiones no contributivas, eran administradas por el mismo sistema y tenían por objeto fundamental asistir a quienes por alguna razón no habían logrado acceder a los beneficios contributivos. En paralelo, el Estado desarrolló una serie de políticas universales en salud y educación no vinculadas al mercado de trabajo, y algunas intervenciones puntuales como programas de salud materno - infantil y de alimentación, entre muchos otros (Cruces \& Rovner 2008).

El hecho que los PTC sitúen el foco en el desarrollo humano de los niños y adolescentes ha hecho que alcancen mayor legitimidad. En esta misma línea, en los últimos años se ha diagramado una estrategia destinada a que figura femenina del hogar sea quien reciba la transferencia monetaria. Ello se produjo bajo la concepción de que ella hará un mejor uso del dinero, a la vez de convertirse en un mecanismo de empoderamiento de la mujer dentro del núcleo familiar debilitando ciertas postulados machistas de los diseños sociales (Banco Mundial 2009).

En 2002, con niveles de desempleo superiores al $20 \%$ y tasas de pobreza superiores al $50 \%$ el gobierno nacional crea el 'Programa Jefes y Jefas de Hogar Desocupados' (PJJHD) que alcanzó a cubrir cerca de dos millones de hogares, equivalentes al $20 \%$ del total del país. Frente a tal situación, en el año 2004, la nueva administración del Presidente Néstor Kirchner adopta una estrategia de transición para los beneficiarios del Plan jefes y jefas de Hogar basada en dos programas: el Seguro de Capacitación y Empleo y el Programa Familias por la Inclusión Social (Gasparini \& Cruces 2008).

Mientras tanto, y en paralelo, como consecuencia de las acciones de movilización que desencadenaron y caracterizaron el estallido del 2001, el Gobierno de la Ciudad puso en 
marcha la distribución masiva de productos alimenticios mediatizada por las organizaciones sociales, fundamentalmente territoriales. Esta intervención consistía en la distribución de alimentos básicos de la canasta familiar a hogares pobres.

La reglamentación del Programa Asistencia Alimentaria Directa a Familias se efectuó recién un día después, al igual que la estandarización de la prestación. Las familias debían reunir requisitos de monoparentalidad, cantidad de hijos con uno o más integrantes en situación de enfermedad grave o desnutrición, con uno o más integrantes con necesidades especiales o bien con uno o más miembros mayores de 65 años que no percibiesen beneficios sociales. (Logiudice 2009: 2)

En el año 2005 se produce la concepción y el lanzamiento del programa 'Ciudadanía Porteña' destinado a reemplazar el programa 'Vale Ciudad', que asumía características similares en tanto se trataba de una transferencia monetaria bancarizada para la adquisición de alimentos en grandes supermercados (Logiudice 2009).

Todos los beneficiarios de los programas 'Vale Ciudad' y 'Apoyo Alimentario Directo a Familias', en tanto calificaran como población beneficiaria, recibirían una prestación de asistencia alimentaria, destinada al hogar, hasta tanto estén en condiciones de ingresar al ‘Programa Ciudadanía Porteña'.

\subsection{La transferencia de Ciudadanía Porteña como activo}

La participación del programa de 'Ciudadanía Porteña', marca una gran diferencia, en sentido positivo, en el nivel de vulnerabilidad de los hogares. El hecho de ser beneficiado con 'la tarjeta', garantiza un ingreso fijo y mensual que permite a los hogares, entre otras cosas, planificar a un mediano plazo y reasignar recursos destinados a la generación de ingresos hacia otro tipo de inversiones como por ejemplo en capital humano -promovido también por la lógica de los PTCs. Su población objetivo son los residentes de la ciudad de Buenos Aires en situación de pobreza y enfatiza su accionar en los de mayor vulnerabilidad, destacando la presencia de embarazadas, menores de 18 años, discapacitados y adultos mayores. La contraprestación de este programa es la asistencia escolar y el control de salud de embarazadas, niños y adolescentes. La titular del beneficio es la mujer, sea esta la jefa de hogar o la cónyuge ${ }^{2}$. La modalidad en la que este realiza las transferencias de ingreso es a través de una tarjeta de compra precargada, que puede ser utilizada únicamente en la red de comercios adheridos al Programa exclusivamente, para la adquisición de alimentos, productos de limpieza e higiene, y combustible para cocinar. Este recurso influye considerablemente en la disminución de la vulnerabilidad de los hogares, dado que constituye un ingreso fijo que pueden complementar tanto con activos que provengan de los otros escenarios de obtención de recursos, como otras 'ayudas sociales' que provengan del Estado.

En el marco de las entrevistas realizadas ${ }^{3}$, fue posible percibir algunos de los usos que las titulares de las tarjetas daban a la transferencia y la forma en la que articulaban el recurso, para optimizar la utilidad de otros activos.

Para mi es una gran ayuda porque es poco lo que puedo hacer yo con mi trabajo y los hijos... y mi mamá es grande y no tiene movilidad, o sea que... es una ayuda, entonces lo que es comida lo cubro... capaz que los gastos extras entonces los puedo cubrir con lo que yo gano. (...) Antes capaz que no compraba todo en un día como lo hago con la tarjeta. Ahora las cosas que ya se que voy a necesitar para

\footnotetext{
${ }^{3}$ Con el objetivo de proteger la identidad de los informantes, sus nombres fueron cambiados.
} 
todo el mes las compro el mismo día, todo junto... Compraba todos los días a medida que iba necesitando. Raquel, 22 años.

Como puede apreciarse, la transferencia cubre básicamente los alimentos que los hogares necesitan consumir y su modalidad permite planificar con los alimentos a largo plazo, dejando el ingreso de capital financiero -dinero en efectivo- que los hogares puedan tener disponibles para ser utilizaros cubriendo otras necesidades.

Con la tarjeta compro Alimentos, galleta, arroz, fideos, cosas de limpieza, pañales. (...) Antes no compraba cosas para limpieza del bebé, sólo pañal. Las carnes también... ahora iba al coto y decía esto quiero, antes no... tenía que cuidarme con la plata. Ahora puedo hacer queque, tortillas, pizza... Sara, 30 años

En muchos casos, la titularidad de la transferencia otorgada a la mujer, acompaña el proceso de su inserción en el mercado de trabajo, dado que dependiendo de la fase del ciclo doméstico en la que se encuentre el hogar, la mujer puede acceder o no a un trabajo de tiempo completo. La situación más recurrente es la participación de las mujeres en trabajos informales e irregulares, que complementan con la transferencia de la tarjeta que les garantiza la alimentación básica del hogar mensualmente. Además, la modalidad de la prestación les permite acceder al beneficio de los descuentos que otorgan algunas cadenas de supermercado a través del pago con débito en determinados días de la semana. Si bien la transferencia del PTC constituye uno de los activos estatales más importantes de los que disponen los hogares, la vivienda también amerita un apartado especial. La titularidad de la vivienda como activo permite a los hogares contar con ventajas diferenciales frente a aquellos que no la tienen.

\subsection{La vivienda social como activo}

La importancia de la vivienda, como un activo productivo para los hogares pobres urbanos, ha sido abordada tanto desde el Asset vulnerability framework, de la mano de Moser, así como también desde la sede de la CEPAL en Montevideo, de la mano de Álvaro Fuentes (1999). Ambas perspectivas coinciden en la importancia de la propiedad de la vivienda, como activo de potencial productividad para los hogares. La misma es identificada como un elemento básico de necesidad o de consumo de los hogares. Sin embargo, es también un importante activo productivo, que amortigua los hogares frente la pobreza extrema. La inseguridad que genera la falta de vivienda, la ocupación de los hogares sin el título formal legal, crea una sensación de vulnerabilidad y en contraposición, la seguridad de tenencia y titulación legal de los hogares brinda el incentivo para invertir en mejoras, y la seguridad de utilizar este recurso de manera productiva, sobre todo cuando las otras fuentes de ingresos son reducidas.

Por otro lado, desde la oficina de la CEPAL en Montevideo, Álvaro Fuentes (1999), afirma que la vivienda constituye uno de los principales activos con que cuentan los hogares. Esto es así en tanto el gasto en vivienda representa una proporción considerable del presupuesto familiar entre los arrendatarios, al mismo tiempo que implica un ahorro igualmente importante cuando se dispone del bien en propiedad o usufructo. En este sentido, las formas de acceso a la vivienda pueden ser examinadas en sus efectos positivos o negativos, dentro del abanico de recursos que movilizan los hogares para el bienestar.

Con respecto al Estado, y pese a su evidente repliegue en muchas áreas, sus estructuras de oportunidades siguen siendo las fuentes más significativas para la acumulación de activos en los estratos populares urbanos, lo que se manifiesta a través de múltiples funciones. 
El Estado es también importante como proveedor directo de activos físicos y en cuestiones vinculadas a los recursos humanos, a través de la asignación de viviendas y de la provisión de educación y salud pública. Por lo tanto, a los fines del análisis concreto de los usos de los activos provenientes del Estado en los hogares estudiados, se tomarán como eje de comparación la posesión y no posesión de la vivienda, como activo diferencial en el proceso de transformación de recursos en activos intercambiables.

\subsection{La transformación de los recursos del Estado en activos}

Recapitulando lo mencionado anteriormente, a la hora de analizar la estructura de oportunidades de los hogares, es importante tomar en cuenta la fase del ciclo doméstico en la que se encuentran dado que los distintos elementos constitutivos del enfoque, varían de acuerdo al ciclo doméstico por el que transitan los hogares. Por lo tanto, tomando en consideración la posesión del activo vivienda y la fase del ciclo doméstico en la que se encuentren, se realizará el análisis de los hogares seleccionados.

El primero de los casos para abordar es el hogar de Tota, quien reside en la villa 20. Es madre soltera y tiene cuatro hijos. Vive en el mismo terreno que la madre, en una casa de construcción precaria, que armó en el patio trasero. Si bien el título de la propiedad de la vivienda en la que reside no es uno de los activos con los que cuenta, al extender la unidad doméstica de su madre no lo percibe como un problema. Este no es el caso de María, quien debe alquilar una vivienda porque no cuenta con familiares propietarios como para acceder a una parcela de tierra como lo hizo Tota.

La situación de María, representa uno de los puntos intermedios en el ciclo doméstico. Si bien ella se encuentra en edad reproductiva y con hijos pequeños (fase expansiva), el hogar de su madre finalizó su fase de dispersión transformándose en unipersonal, razón por la cual ahora reside con ella haciendo uso de su vivienda.

Sin embargo, la vivienda no es el único activo que proviene desde el Estado para asistir a los hogares en situación de pobreza. Tota, por ejemplo, cuenta con un buen racimo de oportunidades que le brinda el Estado, para mantener al grupo doméstico. Por un lado, trabaja en una cooperativa con los Ilamados 'pechitos amarillos' -por el color del uniforme en concordancia con el color político del partido en el gobierno- subvencionada por el Gobierno de la Ciudad y que a su vez presta servicios de saneamiento en el barrio porteño, aunque jurídicamente es una cooperativa independiente. Su estructura de oportunidades está directamente vinculada al Estado, dado que su fuente de trabajo proviene desde el gobierno municipal, así como los otros beneficios económicos que percibe desde las diversas líneas de acción que provienen del Ministerio de Desarrollo Social: la tarjeta de Ciudadanía Porteña y el programa Nuestras Familias.

Ahí en la misma junta me los dieron. Si, como saben que tengo cuatro chicos, los crío yo sola... entonces cada plancito que hay, me dicen "vos necesitas" y obvio... "yo necesito" ja ja [en tono sarcástico]

El caso de Tota muestra cómo la intervención del Estado representa un factor determinante de la estructura de oportunidades de los hogares en situación de pobreza de la ciudad.

No obstante, sí lo es en la vida de María, segundo caso de estudio, quien se vio profundamente afectada por la crisis económica del 2001 cuando vivía en la provincia de Buenos Aires y fue desalojada de su vivienda. La familia estaba dividida en ese momento, su marido estaba trabajando en Catamarca y ella estaba sola con sus hijos, en Buenos Aires. Después del desalojo se alquiló una pieza en la que vivía con sus cinco hijos, en la que pagaba 250 pesos por mes. Posteriormente, consiguió un departamento en 
Piedrabuena por el mismo costo que pagaba la pieza, así que se mudó. El departamento en el que vive está compuesto por cinco habitaciones: tiene un pasillo, dos baños, comedor, cocina y patio.

Pero está destruido. No tiene gas y para que nos pongan la luz, tuvimos que generar un incendio

La vivienda que habita tiene muchas deudas, en todos los servicios que los dueños no pagaron. María inició un trámite de regulación y el trámite para recibir una vivienda del Instituto de Vivienda de la Ciudad de Buenos Aires (IVC):

Pero me dijeron que no hay presupuesto, si hay un censo capaz que me lo dan (...) en la villa cualquiera usurpa un terreno y es de el. Si no hubiera alquilado y me hubiera ido a la villa, ahora tendría un departamento. Es imposible lograr las cosas bien

La ausencia de un título de propiedad del lugar en el que reside con su familia, sumado al costo del alquiler que debe afrontar todos los meses, representa un verdadero problema en su planificación doméstica. A esto debe añadirse el hecho de que la coyuntura económica del hogar es además muy compleja, debido a que cinco meses previos a la entrevista, el desempleo aquejaba al jefe de hogar, originando una readaptación de las actividades económicas ante la crisis.

Los usos del capital físico, como lo es la vivienda, se modifican notoriamente entre las dos ejemplificaciones de los hogares citados, cuando cuentan con una vivienda propia a través de la cual obtendrán un título de propiedad y cuando no. El activo vivienda es muy importante a la hora de disponer del abanico de activos.

Los dos casos que se analizarán a continuación, que se encuentran en la situación descripta, son beneficiarios de una vivienda social a la que accedieron a través del IVC, y residen una en el barrio Pirelli y la otra en los 'monoblocks de Piedrabuena'.

Juana, vive en el barrio Pirelli en una vivienda que acababan de recibir del IVC al momento de la entrevista. Antes vivían en la ex villa 17 - también conocida como Pirelli. A su familia se les había asignado una vivienda siete meses antes de de la entrevista. Se trata de un departamento nuevo, que estrenaron ellos al llegar, con dos habitaciones, una cocina/comedor, un baño y un patio -de reducido espacio.

Me vinieron a hacer un censo, y después ellos mismos seleccionaban a la gente. (...) pago 128 pesos

Es importante recapitular con lo mencionado anteriormente, y destacar el detalle de que ellos accedieron a la vivienda del IVC a través del padre de Nahuel, quien trabaja en esa delegación del Estado e intercedió para facilitarles el acceso a este tipo de capital físico. Con esta afirmación no se trata de mencionar que ellos no constituyeran población objetivo de la vivienda, sino que sus redes familiares fueron de gran ayuda a la hora de acceder a la vivienda de la que hoy disponen.

Además de la vivienda, Juana cuenta con un abanico de activos provenientes del Estado que proviene de diversas líneas de acción. Por un lado es beneficiaria del Programa Ciudadanía Porteña, así como también decidió retomar sus estudios secundarios para poder finalizar la escuela, motivo por el cual es beneficiaria también del componente Estudiar es Trabajar -porque entra en el rango de edad establecida como criterio de focalización.

Asimismo, cuenta con otros beneficios como ser el VIEL y el Nuestras Familias. 
¿Y cuando él no tenía trabajo, como hacías?

Con la tarjeta y casi siempre está un subsidio... que lo anotaba el papa pero...

Esta articulación con los beneficios que el Estado brinda, la obtiene a través de sus redes familiares, específicamente del padre de Nahuel que intercede para que así sea.

Otro caso que ilustra la situación de aquellos hogares que disponen del activo físico que constituye la vivienda, es la unidad doméstica de Clara quien vive en Piedrabuena, en un departamento otorgado por el IVC un par de años antes que el de Juana.

Lo estoy pagando...

¿Lo obtuviste por el IVC?

Si, hace tres años... tres años y medio.

Unos pocos meses antes de la entrevista, el matrimonio había decidido renunciar al ingreso monetario del jefe de hogar para emprender un proyecto conjunto, instalando un kiosco en una de las esquinas del barrio Piedrabuena. Además de la vivienda en la que residen, la transferencia de Ciudadanía Porteña, es el único activo con el que cuentan que proviene del Estado. Como el comienzo de todo negocio, la instalación del kiosco representó una gran inversión de activos, principalmente monetarios, de los que disponía el hogar, renunciando a la retribución inmediata para un beneficio mayor en el largo plazo. Es en este momento, en el que juega un papel crucial la transferencia de Ciudadanía Porteña por que representa un ingreso fijo, el cual por mínimo que sea, es un ingreso garantizado del que disponen. Éste beneficio les permite asumir otros riesgos como ser el afrontar un nuevo negocio.

Aquí es crucial la capacidad de los hogares, y en este escenario de análisis, los activos provenientes del Estado cumplen un rol importante. En este caso en particular, brindó un tejido de seguridad social garantizando la vivienda y el ingreso fijo de una unidad doméstica que vio reducida su vulnerabilidad, y se descubrió en condiciones de afrontar un gran riesgo en vistas de incrementar su bienestar en el futuro a través de una inversión a largo plazo.

Además, otro de los factores que facilitaron esta decisión, es la fase del ciclo doméstico que el hogar transitaba, contando con la mano de obra de sus hijos más grandes, quienes además no requieren de cuidados constantes, permitiéndole contar con más tiempo disponible al matrimonio.

La estructura de oportunidades de los hogares, es muy diferente en cada uno de los casos. Si bien todos cuentan con importantes activos provenientes del Estado, la transformación de los recursos al interior de cada uno de ellos, varía de acuerdo a sus características particulares. A pesar de que el Estado representa un escenario de suma importancia, varía mucho en las diversas formas en las que influye en la estructura de oportunidades, dependiendo de los activos que otorgue.

Catalina Arteaga Aguirre (2003) afirma que en América Latina, a partir de los procesos de reforma del Estado y liberalización de las políticas económicas, "se está transitando de una estructura de oportunidades centrada en el Estado, a una centrada en el mercado y la Sociedad; o donde al menos estas dos instancias de coordinación aparecen perfiladas más claramente junto al debilitamiento de la primera" (Arteaga 2003, citada en Eguía \& Sotelo 2007: 106). 


\section{Las redes de reciprocidad}

En la década de 1970, Lomnitz en su obra "¿Cómo sobreviven los Marginados?" sentenciaba que sobre la precaria base económica de la marginalidad se ha levantado una estructura social específica caracterizada por garantizar la subsistencia mínima durante los períodos más o menos largos e irregulares de la actividad económica. "Esta estructura comporta el predomino de cierto tipo de agrupación: las redes de intercambio entre parientes y vecinos" (Lomnitz 1975: 26). Propone que estas redes representan el mecanismo socioeconómico que viene a suplir la falta de seguridad social, reemplazándola con un tipo de ayuda mutua basado en la reciprocidad. Detecta como el factor determinante de la existencia de los marginados a la condición de inseguridad crónica de empleo y de ingresos, enfatizando en el tipo de intercambio que prevalece en los sectores más vulnerables: el intercambio recíproco que asegura la supervivencia de un importante estrato de la sociedad urbana latinoamericana. Al compartir sus recursos, escasos e intermitentes, con los de otros en idéntica situación, el poblador de los suburbios logra imponerse en grupo a circunstancias que probablemente lo harían sucumbir como individuo aislado (Lomnitz 1975).

Por otro lado, el sociólogo francés Serge Paugam (1991) afirma que los marginados pueden enfrentarse a su condición a través de diferentes actitudes: una de ellas, la actitud de "evitar la marginalidad" -la resistencia a la descalificación, aun cuando el cambio de comportamiento y la identidad parece inevitable- o la "organización en la marginalidad" con la racionalización de la vida diaria, buscando espacios de autonomía.

De estas dos actitudes, se distinguen dos experiencias diferentes de marginalidad vividas. Por un lado, la "marginalidad evitada", que revela los intentos de integración social de aquellos que están tan abrumados por la desgracia que intentan encontrar un equilibrio y poner fin a su vida marginal. Al respecto, Se puede entrever a través de esta experiencia las condiciones necesarias para lograr la promoción social de personas sin status. Por otro lado, se encuentra la "marginalidad organizada", que corresponde a la reconstrucción simbólica de un marco cultural tolerable dentro de un espacio controlado por la experiencia de intercambios y de actividades cotidianas y quizás gracias a los recursos del mundo imaginario. A través del espacio vivido, que contiene potencialmente su historia hecha de conflictos, de fracasos, pero también de fiestas y de momentos felices, ellos acceden a una forma de identidad positiva. Esto no significa una voluntad de cambio de status, sino más bien una adaptación individual a una condición que, se podría decir, está al límite de la exclusión social (Paugam 1991).

Es justamente esta forma de experimentar la marginalidad de forma organizada, la que sirve de disparador para abordar el siguiente análisis sobre la esencia de las redes de reciprocidad.

\subsection{Las redes de intercambio recíproco}

Varios informes publicados a lo largo del tiempo demuestran que las redes sociales han sido los principales medios a partir de los cuales los pobres latinoamericanos han hecho frente a la vida urbana, proporcionando el apoyo y la pericia para construir una casa, encontrar empleo u obtener ayuda en emergencias financieras y médicas (Roberts 1978). Los pobres han demostrado ser hábiles para la creación de redes sociales, particularmente con los parientes (Lomnitz 1975; González de la Rocha 1986).

Las redes están definidas por los criterios de proximidad, distancia social e intercambio de bienes y servicios. El criterio básico es la existencia de una "corriente permanente de

\footnotetext{
${ }^{4}$ Traducción propia: este fragmento ha sido traducido de su versión original "La disqualification sociale: Essai sur la nouvelle pauvrete"
} 
intercambio recíproco" (Lomnitz 1994: 71) desarrollando su trabajo bajo dos elementos claves: la 'cercanía física' y la 'confianza'. Esta última permite entablar un diálogo o intercambio recíproco, crea una voluntad colectiva de cumplir con las deudas o convenios entre las partes, familiaridad suficiente para no ser rechazado.

La red, a su entender, está definida por un sistema de intercambio específico que conlleva a una estructura social de mayor complejidad. La autora hace una diferenciación importante en cuanto a tipos de redes sociales. En primer lugar se encuentra la red egocéntrica, que se refiere al total de individuos con quienes la unidad doméstica intercambia recíprocamente bienes y servicios. En un segundo orden se sitúa la red exocéntrica donde se presenta el intercambio de todos con todos (Lomnitz 1975).

Mercedes González de la Rocha (1986) también aborda - al igual que Lomnitz- la tipología de la red en cuanto a las características relacionales entre los individuos en términos de jerarquía. No obstante, brinda un factor adicional al análisis del comportamiento de los hogares en las redes de intercambio, el ya anteriormente mencionado 'ciclo doméstico'.

La unidad doméstica ofrece la oportunidad de entender mejor el impacto de los procesos "extra domésticos", o más generales sobre la vida familiar - la organización social del mercado de trabajo, los movimientos migratorios, etc. Proporciona asimismo, la oportunidad de entender las fuerzas internas de la unidad doméstica que afectan la organización y la economía familiares y los procesos de toma de decisiones que se desarrollan en su interior. (González de la Rocha 1986: 68)

Las redes constituyen un tipo de activo con el que cuentan las unidades domésticas. Son los contactos sociales y las relaciones, dentro de la clasificación de Filgueira y Katzman (1999), los que denominan "capital social". Éstos pueden considerarse como bienes cuando pueden ser utilizados para conseguir información útil en relación con las fuentes de créditos, trabajos, mercados o servicios para un hogar, como es el caso de una madre que cuida de los hijos de otra que sale a trabajar, por ejemplo.

La cercanía física es uno de los factores que favorecen el intercambio, "a mayor vecindad, mayor interacción social y mayores posibilidades de intercambio" (Lomnitz 1975: 28). No obstante, la confianza es un rasgo cultural que incluye la capacidad y deseo de entablar una relación de intercambio, la voluntad de cumplir con las obligaciones de dicha relación y la familiaridad mutua, como para servir de base a un acercamiento con probabilidad de no ser rechazado. Ambos conceptos se encuentran fuertemente interrelacionados.

\subsection{Reciprocidad y formas de supervivencia organizadas}

Dejando de lado por un momento a las descripciones empíricas, la pregunta que surge al reflexionar sobre esta estructura social específica es cuál es la naturaleza del de intercambio en relación al conjunto del sistema social en el cual se produce. Aunque los intercambios entre los marginados parecen ser más intensos, es necesario profundizar sobre la medida en la que se relacionan en el contexto del sistema social donde sobreviven.

En su obra De la división social del trabajo, Emile Durkheim (2001 [1893]) habla de "reciprocité" pero no de forma conceptual general sino como corolario o atributo de la solidaridad orgánica (Abduca 2007). Se trata de una complementariedad que emerge de la división del trabajo, como el lazo mutuo de obligaciones recíprocas entre el criado y el amo. 
El lazo que, en ese caso, une el individuo al jefe, es idéntico al que en nuestros días liga la cosa a la persona. Las relaciones del déspota bárbaro con sus súbditos, como las del dueño con sus esclavos, del padre de familia romana con sus descendientes, no se distinguen de las del propietario con el objeto que posee. No tienen nada de esta reciprocidad que produce la división del trabajo. (Durkheim 2001 [1893]: 214)

Sin embargo, según Lévi- Strauss (1993 [1949]) los fundamentos de la reciprocidad descansan en los postulados que hiciera Mauss (2010 [1923]) sobre la teoría del "don". Al respecto, aunque no haga referencia directa al concepto "reciprocidad" en su obra, lo utiliza como un adjetivo que refiere a la "generosidad recíproca" sin hablar de "reciprocidad en general".

Marshall Sahlins en su obra Stone Age Economics (1972), intentó analizar este fenómeno social del intercambio retomando el concepto de don definido por Mauss a partir de la noción de contrato jurídico, de tipo más universal, determinando la famosa regla del intercambio que consiste en tres obligaciones fundamentales -dar, recibir y devolverconformando una cadena obligatoria de acciones para que el don funcione socialmente.

\subsection{La continuidad de reciprocidades}

Sobre el sistema de las tres obligaciones del intercambio, Marshall Sahlins (1972) realiza una lectura diferente. Para este autor, el intercambio se puede interpretar como una continuidad de reciprocidades que se caracterizan por la particularidad de su forma.

Cada una de estas formas de reciprocidades expresan relaciones sociales de naturaleza muy distinta, que varía según el contexto y los individuos o grupos implicados en el intercambio. La diversidad de estas formas nos obliga a considerarlas, de manera sistemática y no de manera independiente. Una relación social determinada puede dificultar cierto movimiento de bienes, pero una transacción específica puede - del mismo modo- inducir una relación social particular. Esto es especialmente cierto en el restringido contexto de las transacciones materiales por oposición a un principio social mucho más extendido o a la norma moral de dar y recibir (Sahlins 1972).

La reciprocidad puede establecer relaciones solidarias en la medida en que la corriente material dé idea de beneficio o asistencia mutuos, sin embargo, la realidad social de las partes es ineludible. Es toda una clase de intercambio, un continuo de formas.

\subsection{La reciprocidad generalizada, el extremo solidario}

La reciprocidad generalizada, refiere a transacciones que pueden ser consideradas altruistas, transacciones que están en la línea de la ayuda prestada, y, si es posible y necesario, de la ayuda retribuida aunque no necesariamente. El caso que nos interesa sería un caso típico de 'solidaridad familiar', siempre y cuando la familia tenga con que ayudar al resto del grupo. Es una reciprocidad que está garantizada solo con el hecho de ser miembro de la familia - y la definición de familia puede ser muy amplia- y no se espera que la cadena de reciprocidad se cumpla en plazos cortos. Uno espera simplemente que como miembro de la familia va a encontrar la ayuda que necesita cuando lo sea necesario (Sahlins 1972).

Esto no significa que entregar cosas de esta manera, incluso a los 'seres queridos' no genere una contra obligación. Sin embargo, esa contra obligación no se estipula por tiempo, cantidad o calidad; la expectativa de reciprocidad es indefinida. Por lo general, sucede que el tiempo y el valor de la reciprocidad no sólo dependen de lo que el dador ha entregado, sino también de lo que éste pueda necesitar y del momento en que lo necesite, y del mismo modo de lo que el receptor puede pagar y cuándo puede hacerlo. 
La reciprocidad generalizada basada en el vínculo familiar puede observarse concretamente, en el hogar de Tota, vinculado a la vivienda en la que reside. Ante la falta de un lugar para habitar, recurrió a su red familiar, la propiedad de su madre. Sin embargo es importante tener en cuenta que esta red, contempla un integrante más, Julieta, la hermana de Tota.

Julieta también es madre soltera y vive con sus cinco hijos. Su matrimonio se disolvió a raíz de una serie de episodios de violencia, por lo que no tiene contacto alguno con el padre de sus hijos. Si bien Julieta no reside en el terreno de su madre como Tota, requiere de la ayuda de su madre para el cuidado de sus hijos mientras trabaja.

No, los míos están todo el día acá jugando a la pelota [mientras señala el patio común entre las casas de Tota y su madre] (...) los cuida la abuela

Ante la ruptura del vínculo con el padre de sus hijos, Julieta se vio obligada a ingresar al mercado laboral, para la generación de activos financieros, aunque para ese entonces ya contaba con la tarjeta de Ciudadanía Porteña. El trabajo, lo consiguió a través de sus redes de amistades, ya que una amiga -la misma amiga que llevó a Ana al mercado de trabajola recomendó para un trabajo de obrera en la fundación que trabaja.

Si Julieta no hubiera contado con la contención de su red familiar, no hubiera podido aceptar el trabajo de tiempo completo del que participa actualmente, porque no tendría con quien dejar a sus hijos de temprana edad. No obstante, esta situación cambia en la situación de Tota. Si bien ella también tiene un trabajo que demanda de su presencia todo el día, no demanda de ese favor específico de su madre, porque reside en el mismo terreno que ella.

¿Y los chicos a parte de ir a la escuela, hacen alguna actividad?

No, sólo van a la escuela. El nene de 14 si los sábados va a jugar a la pelota. Los más chiquitos no.

¿Vienen acá también, a lo de la abuela como los hijos de Julieta?

Sí, pero ellos no son tan "abueleros" (...) les gusta estar mirando tele en casa.

¿Y no los cuida nadie?

No, a ellos no los cuida nada. Igual, si pasa algo, la abuela está, las tías también, pero yo no le dejo la responsabilidad a ellos "cuídamelos". No, yo me voy, ya bastante tiene con todos los chicos de Juli, los míos que se cuiden solos.

Si bien las dos hermanas tienen en común a su madre en el marco de la red familiar de la que participan, una de ellas hace uso del capital físico de su madre y la otra, hace uso de su capital humano. Sin embargo, se evidencia en el testimonio de Tota que la solicitud de servicios de la madre necesita moderación. Al ser ya beneficiaria del terreno para su residencia, evita solicitar de su madre el cuidado de sus hijos, mientras que Julieta - que alquila una vivienda a unas cuadras de la casa de su madre- deja a sus hijos a su cuidado durante todo el tiempo que se encuentre en el trabajo. Si bien la retribución no es inmediata, se toman ciertos cuidados a la hora de hacer uso del vínculo familiar para la solicitud de favores y servicios.

\subsection{La reciprocidad equilibrada, el punto medio}

Se refiere al intercambio directo. En un equilibrio preciso la reciprocidad consiste en la entrega habitual del equivalente de la cosa recibida sin demoras. Puede aplicarse con más aptitud a las transacciones que estipulan una retribución de valor o utilidad conmensurados dentro de un período finito y no muy largo. Las partes se enfrentan como intereses económicos y sociales distintos. El aspecto material de la transacción es, por lo 
menos, tan importante como el social; hay un reconocimiento más o menos preciso, ya que las cosas dadas deben ser retribuidas dentro de un corto período. La retribución debe darse en un plazo de tiempo definido. La gente actúa adherida a intereses económicos y sociales separados (Sahlins 1972).

Además de los parientes, los vecinos también son fuente de préstamos, ya sean pequeños para hacer frente a dificultades de la vida cotidiana, o más importantes, si se tiene noticia de que un vecino ha tenido una entrada importante de dinero. En el primer caso, circulan sumas muy pequeñas, prestadas sin interés por un periodo muy corto. En el caso de una solicitud importante de dinero, se entra en el sistema de préstamo con interés. La sanción al mal pagador, tanto en el caso de préstamos elevados como en el de reducidos, viene con cierta rapidez. "La fuente de préstamo se cierra para él. Debe entonces, dirigirse a otras instancias. Si continúa haciendo varias veces lo mismo en el barrio, su reputación queda manchada muy rápidamente y nadie le querrá hacer un favor" (Chamoux 1993: 200).

En el caso de la amistad, el préstamo debe efectuarse en el marco de legitimidad definida en términos locales, para que los medios habituales de control, y sobre todo la publicidad puedan funcionar. El riesgo es particularmente grande cuando el control descansa solo en relaciones interindividuales. En este sentido, el hogar de Julieta, muestra que ante la coyuntura en la que vio su matrimonio disuelto y se encontró sola con cinco hijos, una amiga la recomendó en su trabajo para que pudiera acceder a un ingreso fijo, si bien no constituye un préstamo de dinero en efectivo, la recomendación de un amigo en un trabajo requiere de una profunda confianza, dado que una mala recomendación podría resultar en la pérdida de la fuente laboral para ambas personas.

Otro ejemplo de este tipo de reciprocidad, retomando la ejemplificación del hogar de María, es la solidaridad con los vecinos. Ante la condición de desempleo del marido de María y la llegada de la crisis con los cinco meses del cese de actividades, María decidió ingresar al mercado laboral. Dadas las dificultades que afrontaba el hogar los vecinos de la familia, decidieron solicitar un servicio de María que permitiera otorgarle una ayuda 'digna' sin caer en el endeudamiento. Por este motivo, comenzó a limpiar las escaleras del edificio por 20 pesos al día.

Este tipo de 'changuita' le permite mantener sus actividades domésticas en el hogar y no caer en el endeudamiento solicitando préstamos de sus redes de familiares y amigos. Sin embargo, su participación en la actividad económica, fue una medida para resolver una coyuntura particular, por lo que al recuperar Roberto su trabajo, María dejó de hacer la limpieza de las escaleras del edificio, pues la necesidad ya no era inmediata.

\subsection{La Reciprocidad negativa, el extremo insociable}

Es el intento de obtener algo a cambio de nada gozando de impunidad; entran aquí las distintas formas de apropiación, las transacciones iniciadas y dirigidas en vistas a una ventaja utilitaria neta. La reciprocidad negativa es la forma más impersonal de intercambio. Los participantes se enfrentan como intereses opuestos, tratando cada uno de obtener el máximo de utilidad a expensas del otro. Considerando la transacción con vistas al provecho propio, la parte que inicia el trato, o ambas partes, se proponen lograr un incremento no ganado. Desde aquí, la reciprocidad negativa pasa por todos los matices que van desde la astucia, la ingeniosidad, las artimañas y la violencia. Se trata de la defensa del propio interés (Sahlins 1972).

Es una relación en la cual se trata de obtener un beneficio a expensas de la otra parte. Incluye al regateo, la trampa y el robo. Los participantes tienen una relación estructural social distante, presentan intereses opuestos y buscan maximizar su beneficio. En todo caso, es una relación entre grupos distantes y simétricos. 
Dentro de los hogares analizados a los fines de esta investigación, se puede desarrollar un ejemplo de las modalidades de reciprocidad coexistentes en el marco de una misma red, de la que participan algunos hogares entrevistados.

El hogar de Graciela está compuesto por ella, su marido y su hija Niki. Sin embargo, para ser beneficiaria del programa Ciudadanía Porteña, recurrió a la trampa y declaró una unidad doméstica en la que ella es jefe de hogar y no tiene marido. Además, dadas las características del PTC -el monto es calculado en base a los miembros del hogar- declaró que residen con ella, sus otros hijos, que ya conformaron unidades domésticas aparte. Esta artimaña, es una de las trampas características de la reciprocidad negativa, en las que una de las partes busca maximizar sus beneficios a expensas del otro. A través de su falso testimonio, se presenta ante la agencia estatal que otorga el beneficio como un hogar en situación de vulnerabilidad mayor para acceder a una transferencia mayor.

No obstante, la trampa no finaliza en la declaración fraudulenta de la composición de su hogar, sino que otra de las características distintivas de los programas sociales de la ciudad de Buenos Aires - tanto Ciudadanía Porteña como los otros de los que es beneficiaria- es que ningún miembro del hogar puede ser empleado del Gobierno de la Ciudad de Buenos Aires. Como fue mencionado anteriormente, Cesar, el marido de Graciela, no sólo es empleado del Gobierno de la Ciudad, sino que participa de una institución clave de distribución de activos del Estado, el Instituto de la Vivienda (IVC).

A través de las artimañas del matrimonio, fueron todos sus hijos beneficiarios de una vivienda - reciprocidad generalizada- de las últimas entregadas por dicha entidad, así como también, son beneficiarios de la mayoría de los programas sociales que van saliendo, debido a sus influencias. Uno de los hogares beneficiados directamente por esta situación, es la anteriormente desarrollada unidad doméstica de Juana y Nahuel.

Se puede percibir la asimetría del hogar, específicamente del matrimonio en su relación con el Estado. Sin embargo, esta situación no se limita a dicha jurisdicción. El engaño y la búsqueda del propio provecho se extienden a sus redes de amigos y vecinos. A través de un diálogo con la gente del barrio Pirelli fue posible reconstruir, a través de una serie de testimonios, que además de sacar provecho de su relación con el Estado, Cesar hacía uso de su posición en el Gobierno de la Ciudad, para 'favorecer' a los vecinos y amigos (reciprocidad equilibrada) a ingresar su solicitud a créditos del IVC, cobrando una comisión de 600 pesos argentinos - que transformaba en ingreso para el hogar- tan solo por el 'favor' de interceder por ellos, sin garantía de resultados.

Otro ejemplo de estas artimañas fue explicado por Tota en una entrevista. Ella no se ve directamente involucrada con el tipo de reciprocidad negativa en su descripción, pero es de utilidad a modo de ejemplo:

Ta' bien, hay mucha gente que vive acá en la villa por comodidad. No pagás, edificás a cuanto querés, lo das todo en alquiler. Tenés almacén y no nada porque se sabe que los que tienen almacén fuera de la villa tienen que pagar monotributo, DGI y miles de cosas. Acá las tienen a los almacenes y no pagan nada, tienen talleres de costura y no pagan un solo centavo. O sea, es más rentable la villa. Convengamos que es más rentable la villa para el que sabe hacer sus negocios, ¿no? Pero también hay mucha gente que lo necesita porque no tiene otra cosa...

Una consecuencia característica de la reciprocidad, es la elaboración de un código moral diferente - y a veces opuesto- al código moral del intercambio de mercado. En una relación de reciprocidad, existe un énfasis moral explícito en el acto de dar, o de devolver el favor recibido, antes que de extraer el máximo beneficio inmediato de una transacción. 
Ambos sistemas pueden utilizarse paralelamente en diferentes contextos, puesto que un miembro de una red de intercambio recíproco puede simultáneamente vender sus servicios o su fuerza de trabajo en el mercado laboral. Pero en último término es la reciprocidad con sus parientes y amigos, la que asegura su supervivencia entre los largos y frecuentes intervalos de cesantía, a pesar de que el mercado proporcione todos los recursos económicos. Cuando estos recursos no van acompañados de un mínimo de seguridad, no logran garantizar el sustento. En cambio, si estos recursos intermitentes de comparten entre seis, ocho o diez personas, el grupo sobrevivirá allí donde sucumbirá el individuo (Lomnitz 1994: 91- 92).

\subsection{La vulnerabilidad de los hogares}

Hasta aquí se han analizado los tres escenarios de los que provienen los recursos que articulan y transforman los hogares en situación de pobreza, ejemplificando con la situación real a la que se enfrentan los hogares de Villa Lugano. No obstante, para comprender el desajuste entre los activos y la estructura de oportunidades que plantea el enfoque AVEO, es necesario tener en cuenta que "los cambios en la vulnerabilidad de los hogares pueden producirse por cambios en los recursos que poseen o controlan, por cambios en los requerimientos de acceso a la estructura de oportunidades de su medio o por cambios en ambas dimensiones" (Filgueira \& Katzman 1999: 8). La vulnerabilidad social no es ni activo ni estructura de oportunidades sino la intersección entre ambos (Filgueira 2001).

Existen variables de gran importancia que afectan el modo en el que los hogares reaccionan al cambio social y económico a gran escala. Estas variables son relativamente autónomas y se derivan de algunas características intrínsecas de los hogares y del modo en el que los hogares evolucionan con el tiempo. Su capacidad para combinar los recursos y convertirlos en bienes variará de acuerdo con los factores como lo son la composición y el tipo de hogar, su tamaño, la jefatura del hogar, la etapa del ciclo doméstico y otros factores que determinan el número de contribuyentes potenciales a la economía del hogar (González de la Rocha \& Escobar Latapí 2006: 69).

\section{Conclusiones}

Para analizar las modificaciones que un PTC como Ciudadanía Porteña realiza en el abanico de activos de los hogares beneficiarios de Villa Lugano, el enfoque seleccionado permitió, llevar a cabo un abordaje integral de la situación particular de cada uno de los hogares observados focalizando en el desajuste entre los activos y la estructura de oportunidades que configuran el mercado, el Estado y la sociedad. Estos tres elementos influyen de manera determinante en las capacidades de los hogares de mantener o incrementar su bienestar, dado que constituyen el escenario donde se generan los recursos.

El mercado de trabajo como principal fuente de ingresos monetarios, al no poder movilizarse, llevó a los hogares a afrontar cambios en la distribución de recursos a su interior para prevenir que su capacidad de supervivencia se erosione. Ante las fluctuaciones cíclicas de la economía local, los hogares - dependiendo de sus características particulares- respondieron a las crisis con medidas de adaptación al contexto, basadas principalmente en el incremento de trabajadores asalariados generadores de capital financiero. En este sentido, la transferencia de Ciudadanía Porteña cumplió un rol de suma relevancia dado que permitió a las mujeres de los hogares, asegurar de forma mensual la alimentación básica de la familia, pudiendo complementar el activo de la tarjeta con una participación informal e irregular en el mercado de trabajo, principalmente aquellas en la fase expansiva del ciclo. 
Por su parte, el Estado representa uno de los escenarios que más influyen en la vulnerabilidad de los hogares: no sólo desde su rol regulador del mercado, sino también como asignador de recursos y como fuente de empleo. Las diversas líneas de acción que benefician directamente a los hogares en situación de pobreza de la ciudad de Buenos Aires, marcan una gran diferencia en el proceso de su supervivencia. Principalmente, el PTC Ciudadanía Porteña, que desde 2005 los asiste mensualmente con una transferencia mensual que les permite contar con un ingreso mínimo y combinable con otros activos que, dependiendo de cada hogar y sus características intrínsecas, favorece a la planificación a un mediano plazo.

Sin embargo, otro recurso de importancia proveniente del Estado que debe considerarse es la vivienda social. Ésta modifica la estructura de oportunidades significativamente, brindando estabilidad y un mayor margen de maniobra para aquellos que son beneficiarios de la misma y disminuyendo las posibilidades de asumir riesgos financieros de aquellas unidades domésticas que no la recibieron.

Por último la sociedad es el espacio en el que los hogares logran intercambiar muchos de los activos no financieros, explotando aquel recurso intangible con el que cuentan cotidianamente: sus redes sociales. La transformación de recursos en activos se lleva a cabo en el marco de las redes de intercambio recíproco en la que participan los hogares, permitiéndoles optimizar sus capacidades de supervivencia. La reciprocidad que las caracteriza puede adoptar diversas modalidades a lo largo de un amplio espectro que define Sahlins (1972) entre la generalizada y la negativa, que pueden darse de forma simultánea en el marco de una misma red de intercambio recíproco en la que participen los hogares. Cada tipo de reciprocidad caracteriza un lazo entre miembros de los hogares, con otros participantes de la red. En este sentido, Ciudadanía Porteña, ayuda a fortalecer algunos vínculos de reciprocidad entre hogares e individuos, principalmente al transformarlos en posibles actores 'fiables' -con capacidad de una respuesta económicaa la hora de solicitar favores ante circunstancias imprevistas.

En este marco, los tres escenarios analizados a partir del enfoque de Activos, Vulnerabilidad y Estructura de Oportunidades, si bien permiten realizar un análisis integral de la situación de cada uno de los hogares, no profundizan su mirada sobre un factor que considero determinante en las capacidades de supervivencia de los hogares: la fase del ciclo doméstico en la que se encuentren los hogares. Ésta última representa la principal fuente de variación no sólo en el grado de intensidad del trabajo, sino de los patrones de consumo. Las etapas por las que van transitando originan una variación significa en la distribución de los recursos.

Los tres momentos que enfrentan los hogares en su ciclo de vida, muestran fortalezas y debilidades. De los casos concretamente analizados a lo largo de esta artículo, se puede afirmar que los hogares en la fase expansiva se encuentran más expuestos a la vulnerabilidad, en relación a aquellos que ya se encuentran en la fase de consolidación, con hijos en edad laboral que no demandan de tiempo de cuidado, ni una asignación exclusiva de activos, ya que de alguna forma se 'independizan' aunque no colaboren con la economía doméstica. La ilustración de hogar en la fase de dispersión que fue analizado, no muestra verdaderamente la realidad de los hogares en proceso de disolución. No obstante, es importante tener en cuenta que se trata de un momento de alta vulnerabilidad, sobre todo cuando se disuelve en núcleo conyugal por fallecimiento y los hogares unipersonales terminan por anexarse a los de sus hijos en las fases dispersión y consolidación, aumentando su exposición a la vulnerabilidad.

Luego de haber analizado la situación de hogares beneficiarios de Ciudadanía Porteña, en el marco de esta investigación, puede afirmarse que la transferencia del programa realiza grandes modificaciones en las capacidades de los hogares analizados, aunque es importante tomar en consideración que no es la única 'ayuda' que reciben desde el Estado 
y que es importante analizarla desde la integralidad del hogar. Las decisiones que toman los hogares en términos de administración de recursos para garantizar su supervivencia, están determinadas, en gran medida, por las variaciones en el mercado de trabajo, la seguridad que garantice la contención del Estado de bienestar y los vínculos de reciprocidad que puedan establecer en sus redes de intercambio recíproco.RM

\section{Bibliografía}

Abduca, R. G. (2007). La reciprocidad y el don no son la misma cosa. Cuadernos de Antropología Social, 26, 107- 124.

Bastagli, F. (2011). From Social Safety Net to Social Policy? The Role of Conditional Cash Transfers in Welfare State in Latin America. International Poverty Center, Working Paper, 60. Consultado el 19 de agosto, 2012, desde http://www.ipcundp.org/pub/IPCWorkingPaper60.pdf

Chamoux, M. N. (1993). Las artimañas del prestamista y del prestatario: los problemas que plantea el crédito invisible. En. M. N. Chamoux et al. (Coords.), Prestar y pedir prestado: relaciones sociales y crédito en México del siglo XVI al XX (pp. 191-210). México D. F.: CIESAS, CEMCA

Cruces, G. \& Rovner, H. (2008). Los programas sociales en la opinión pública. En, Los programas sociales en Argentina hacia el Bicentenario: visiones y perspectivas (pp. 49-120). Buenos Aires: Banco Mundial.

Durkheim, É. (2001 [1893]). La división social del trabajo. Madrid: Akal.

Eguía, A. \& Sotelo, L. (2007). Los programas sociales como recursos para la reproducción familiar. En, A. Eguía, \& S. Ortale (Eds.), Los significados de la pobreza (pp. 85-106). Buenos Aires: Biblos Sociedad.

Filgueira, C. (1998). Welfare and Citizenship: Old and New Vulnerabilities. En V. Tokman, \& G. O'Donnell (Eds.), Poverty and Inequality in Latin America: Issues and New Challenges (pp. 119-139). Indiana, Universidad de Notre Dame.

Filgueira, C. (2001). Estructura de oportunidades y vulnerabilidad social: aproximaciones conceptuales recientes. Seminario Internacional: Las diferentes formas de expresión de la vulnerabilidad social en América Latina. Montevideo: CEPAL.

Filgueira, C. \& Katzman, R. (1999). Marco conceptual sobre activos, vulnerabilidad y estructura de oportunidades. Montevideo: CEPAL.

Gasparini, L. \& Cruces, G. (2008). Programas Sociales en Argentina: alternativas para la ampliación de la cobertura. La Plata: CEDLAS.

González de la Rocha, M. (1986). Los recursos de la pobreza. Familias de bajos ingresos de Guadalajara. México D.F.: CIESAS.

González de la Rocha, M. \& Escobar Latapí, A. (2006). Familia, Trabajo Y Sociedad: El Caso De México. En E. De la Garza Toledo (Ed.), Teorías sociales y estudios del trabajo: nuevos enfoques. México D.F.: Anthropos.

Isuani, A. E. (2008). La política social argentina en perspectiva. En, Los programas sociales en Argentina hacia el Bicentenario: visiones y perspectivas. Buenos Aires: Banco Mundial.

Katzman, R. \& Filgueira, F. (2006). Las normas como bien público y como bien privado: reflexiones en las fronteras del enfoque AVEO. Centro de Investigación Social Un techo para Chile, 41- 70.

Katzman, R., Beccaria, L., Filgueira, F., Golbert, L. \& Kessler, G. (1998). Vulnerabilidad, Activos y Exclusión Social en Argentina y Uruguay. Documento de Trabajo, 107. Santiago de Chile: OIT.

Lévi-Strauss, C. (1993 [1949]). Las estructuras elementales del parentesco. Madrid: Paidós.

Logiudice, A. G. (2009). La asistencia social en la Posconvertibilidad ¿Cambio de paradigma? Algunas consideraciones a partir de los programas locales y nacionales implementados en la Ciudad. Margen, 54. Consultado el 19 de Agosto, 2012, desde http:// www. margen.org/ suscri/ margen54/logiudice.pdf 
Lomnitz, L. (1975). ¿Cómo sobreviven los Marginados? México D.F.: Siglo XXI.

Lomnitz, L. (1994). Redes Sociales, cultura y poder: ensayos de antropología latinoamericana. México D.F.: Grupo Editorial Miguel Ángel Porrua.

Mauss, M. (2010 [1923]). Ensayo sobre el don. Razón y forma del cambio en las sociedades primitivas. Madrid: Katz.

Moreno, J. M. (2008). ¿Ud. volvería a lanzar el Plan Jefes? Una visión de los planes sociales y el mercado de trabajo en Argentina. En, Los programas sociales en Argentina hacia el bicentenario: visiones y perspectivas (pp. 273- 306). Buenos Aires: Banco Mundial.

Moser, C. (1996). Situaciones críticas: Reacción de las familias de cuatro comunidades urbanas pobres ante la vulnerabilidad y la pobreza. Washington D.C.: Banco Mundial.

Moser, C. \& Holland, J. (1997). Urban poverty and violence in Jamaica. Washington DC: Banco Mundial.

Paugam, S. (1991). La disqualification sociale. Paris: PUF.

Sahlins, M. (1972). Economía de la Edad de Piedra. Madrid: Akal.

Unidad de Información, Monitoreo y Evaluación - UIMyE (2009) Mercado de trabajo en la ciudad de Buenos Aires. Serie de informes sobre la situación social de la Ciudad, Gobierno de la Ciudad de Buenos Aires. Consultado el 19 de Agosto, 2012, desde http:// estatico.buenosaires.gov.ar/areas/des social/ evaluacion_programas/informes _condiciones_vida/Mercado_de_Trabajo_2008.pdf

\section{Sobre la autora}

Helga Fourcade se desempeña actualmente como becaria doctoral FONCyT sobre el tema Desarrollo Humano y Social de la infancia en el Observatorio de la Deuda Social Argentina, perteneciente a la Pontificia Universidad Católica de Argentina. Magíster en Antropología Social y Diploma Superior en Antropología Social y Política de la Facultad Latinoamericana de Ciencias Sociales (FLACSO), Argentina. Entre sus últimas publicaciones se cuentan: Qué opinan los niños/as y adolescentes sobre los derechos de los niños (2012) y La mirada de los niños/as y adolescentes sobre su vivienda y su barrio (2012).

helgafourcade@gmail.com

\section{Contacto}

Observatorio de la Deuda Social Argentina

Pontificia Universidad Católica de Argentina

Av. Alicia Moreau de Justo 1500 Edificio San Alberto Magno, 4ㅇ piso, oficina 462

Buenos Aires, Argentina

Recibido: Junio 2012

Aceptado: Agosto 2012 